\title{
Thermo-Magnetic Signature of a Superconducting Multi-band Square with Rough Surface
}

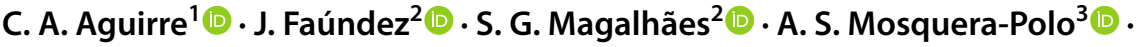 \\ J. Barba-Ortega ${ }^{4}($ )
}

Received: 7 April 2021 / Accepted: 11 May 2021 / Published online: 7 June 2021

(C) The Author(s), under exclusive licence to Springer Science+Business Media, LLC, part of Springer Nature 2021

\begin{abstract}
In the present work, we will study the effect that the surface roughness of the sample has on the magnetic and thermodynamic properties in a mesoscopic superconducting meso-square under an external magnetic field in a zero-field cooling process. We will analyze the magnetization, superconducting electronic density, free Gibbs energy, specific heat and entropy as a function of the roughness of the sample in a superconducting two-band square taking a Josephson type inter-band coupling. We show that the magnetic and thermodynamic properties depend on the roughness percentage of its surface. Our investigation was carried out by numerically solving the two-band time-dependent Ginzburg-Landau equations.
\end{abstract}

Keywords Rough $\cdot$ Superconductivity $\cdot$ Two bands $\cdot$ Calorimetrics

\section{Introduction}

It has been demonstrated that the topology of the surface modifies the properties of the mesoscopic superconductor [1]. A lot of works have been developed taking into account different types of topology and boundary conditions for the vortex state analysis [1-8]. Baelus et al. [9] investigated the influence of surface defects on the vortex penetration and expulsion in thin circular, square and rectangular samples, they found that due to the vortex-vortex repulsion and the vortex-defect interaction, the vortex does not enter or leave the sample through the surface defect. The

C. A. Aguirre

cristian@fisica.ufmt.br

1 Departamento de Física, Universidade Federal de Mato-Grosso, Cuiabá, Brasil

2 Instituto de Fisica, Universidade Federal do Rio Grande do Sul, Porto Alegre 91501-970, Brazil

3 Facultad de Ingeniería, Universidad del Magdalena, Santa Marta, Colombia

4 Departamento de Física, Universidad Nacional de Colombia, Bogotá, Colombia 
effect of a triangular arrangement of punctual defects on vortex configuration in a thin circular mesoscopic sample was done [10], they found non-commensurate vortex configurations due to the interplay between the vortex-vortex repulsion, the vortex-defect interaction and the interaction with the sample border. Numerical simulation of transport characteristic of a mesoscopic superconducting strip with randomly placed pinning centers and taking into account the Bean-Livingston barrier at the edges in an external magnetic field was realized [11]. Experimental and theoretical studies show that at small magnetic fields, there is a discontinuity in heat capacity at critical temperature $\left(T_{c}\right)$ as the bulk superconductors. The heat capacity jump at $T_{c}$ exhibits modulation as a function of magnetic field associated with the known Little-Parks effect [12-17]. In general, a superconductor is a diamagnetic material, but in some special cases and conditions, it can exhibit a paramagnetic Meissner effect (PME). Meyer et al. studied the PME in a small thin ringlike mesoscopic superconductor, the region of PME is above the transition between states of the different angular moments and is well known, this phenomenon is a manifestation of the decreasing energy as the magnetic field is increasing [18]. Palacios et al. explain the PME as a result of the metastability of the vorticity [19]. For high vorticity states, the heat capacity presents a peak whose position with the change in the field is related to the paramagnetic Meiss effect and can lead to an observation of positive magnetization [12]. Huang et al. [20] studied the effects of vacancy cluster defect in the entropy, enthalpy, free energy and heat capacity of silicon crystals, they found that the heat capacity decreases as the vacancy cluster defect size increased. Analytic calculation and a model that describes the low-temperature heat capacity of in-homogeneous cuprates compounds was performed in a mesoscopic disordered $s$-wave superconductor and the results reproduce the features of the heat capacity for $M g B_{2}$ [21-23]. Y. Kleeorin et al. proposed a method to measure the entropy of mesoscopic systems via thermoelectric transport, they proved analytically and demonstrated numerically the applicability of their method [24]. Although there are numerous works on the thermodynamic properties in mesoscopic superconductors, there are still few studies of these properties taking into account the surface defects. In this work, we study the calorimetric properties of mesoscopic superconducting squares with rough surfaces. This paper is organized as follows. The theoretical formalism is presented in Sect. 2. In Sect. 3, we show and discuss the results for heat capacity, magnetization, Gibbs free energy, entropy and vortex states in a superconducting sample with one and two bands with a surface rough and external magnetic field. Finally, in Sect. 4, we detail the main results, including a comparison between the thermodynamic signatures and magnetic properties in a one and two-band superconducting sample.

\section{Theoretical Formalism}

In this work, we investigated the electronic and thermodynamics properties for a superconducting nano-square, in a zero-field cooling (ZFC) process, immersed in an external magnetic field $H$. In dimensionless units, the Gibbs energy functional 
for a two-band superconducting sample taking a Josephson type inter-band coupling is [25-29](and references therein):

$$
\begin{aligned}
\mathcal{G}=\int & \mathrm{d} V\left\{\sum _ { i = 1 , 2 } \left[\alpha_{i}\left|\psi_{i}\right|^{2}+\frac{\beta_{i}}{2}\left|\psi_{i}\right|^{4}\right.\right. \\
& \left.+\frac{1}{2 m_{i}}\left|(-i \hbar \nabla-2 e \mathbf{A}) \psi_{i}\right|^{2}\right] \\
& \left.+\frac{1}{2 \mu_{0}}(\nabla \times \mathbf{A})^{2}+\gamma\left(\psi_{1}^{*} \psi_{2}+\psi_{1} \psi_{2}^{*}\right)\right\},
\end{aligned}
$$

Here $\alpha_{i}, \beta_{i}$ are phenomenological parameters and the last term describes the Josephson coupling, respectively, $\alpha_{i},=\alpha_{i 0}\left(1-T / T_{c i}\right)$ in Eq. (1). so, in Eqs. (2) and (3), $\tau(x, y)=\left(T_{c}(x, y)-T\right) /\left(T_{c 1}-T\right), T_{c 1}$ is the nominal critical temperature of the sample, and $\tau(x, y)$ is a spacial random function that simulates the roughness of the sample. With $\gamma=0$, we obtain the single-band system. In equilibrium, the free energy has a minimum with respect to $\psi_{i}^{*}$ and $\mathbf{A}$, which in London gauge $\nabla \cdot \mathbf{A}=0$, after the dimensionless form, gives:

$$
\begin{aligned}
\frac{\partial \psi_{1}}{\partial t}= & \left(\tau(x, y)-T-\left|\psi_{1}\right|^{2}\right) \psi_{1}-(-i \nabla-\mathbf{A})^{2} \psi_{1} \\
& +\tilde{\gamma}_{J} \psi_{2} \\
\frac{\partial \psi_{2}}{\partial t}= & \left(\tau(x, y)-\frac{T}{T_{r}}-\left|\psi_{2}\right|^{2}\right) \psi_{2} \\
& -m_{r} \alpha_{r}(-i \nabla-\mathbf{A})^{2} \psi_{2}+\frac{\alpha_{r}^{2}}{\beta_{r}} \tilde{\gamma}_{J} \psi_{1} \\
\frac{\partial \mathbf{A}}{\partial t}= & \Re\left[\psi_{1}(i \nabla-\mathbf{A}) \psi_{1}^{*}\right] \\
& +\Re\left[m_{r} \frac{\beta_{r}}{\alpha_{r}} \psi_{2}(i \nabla-\mathbf{A}) \psi_{2}^{*}\right]+\kappa^{2} \triangle \mathbf{A}
\end{aligned}
$$

we defined $m_{r}=m_{1} / m_{2}=0.5, \quad T_{r}=T_{2} / T_{1}=1.0, \quad \alpha_{r}=\alpha_{10} / \alpha_{20}=1.0$, $\beta_{r}=\beta_{1} \beta 2=0.7, \tilde{\gamma}_{J}=\left(\gamma \psi_{20}\right) /\left(\alpha_{10} / \psi_{10}\right)$. We express the temperature $T$ in units of the critical temperature $T_{c 1}$, length in units of the coherence length $\xi_{10}=\hbar / \sqrt{-2 m_{1} \alpha_{10}}$, the order parameters in units of $\psi_{i 0}=\sqrt{-\alpha_{i 0} / \beta_{i}}, \kappa=4.2$, simulating a $M g B_{2}$ sample [30], we choose the zero-scalar potential gauge, $\phi=0$ at all times. Finally, for convergence rule for time:

$$
\Delta t \leq \min \left\{\frac{\mu^{2}}{4}, \frac{\beta \mu^{2}}{4 \kappa^{2}}\right\} ; \quad \mu^{2}=\frac{2}{\frac{1}{\delta x^{2}}+\frac{1}{\delta y^{2}}}
$$

Time is in units of the Ginzburg-Landau characteristic time $t_{G L}=\pi \hbar / 8 k_{B} T_{c} u$, and the vector potential $\mathbf{A}$ is scaled by $H_{c 2}(0) \xi(0)$, where $H_{c 2}$ is the bulk upper 
critical field. Gibbs free energy $G$ in $G_{0}=H_{c}^{2}(0) c h / 4 \pi e \xi^{2}(0)$ units. The entropy $S$ is calculated as $S=\partial G / \partial T$ in units of $S_{0}=\alpha^{2}(0) T_{C} / \beta$, and the specific heat $C=T(\partial S / \partial T)$ in units of $C_{0}=H_{c}^{2}(0) V / 8 \pi T_{c}$. We use Neumann boundary conditions $\mathbf{n} \cdot(i \boldsymbol{\nabla}+\mathbf{A}) \psi=0$. The size of the computational lattice is $N_{x} \times N_{y}$, with $d_{x}=d_{y}=0.1 \xi$ and $N_{x}=N_{y}=200$. TDGL equations are solved using the method of the link variables or $\Psi-U$ method. In this scheme, the order parameter, the vector potential and the external magnetic field are arranged in a discrete lattice bound by the variables $U_{x}$ and $U_{y}$. This method has the important characteristic of preserving the gauge invariance of Eqs. (2)-(4) does not change under the transformation $\psi^{\prime}=\psi \exp (i \chi), \mathbf{A}^{\prime}=\mathbf{A}+\nabla \chi, \phi \prime=\phi-\dot{\chi}[31-35]$ (and reference therein).

\section{Results and Discussion}

In this section, we present the magnetization, heat capacity, vortex states in a superconducting mesoscopic square with surface roughness, both for a single and twoband system. The sample scheme is presented in Fig. 1.

\subsection{Single-Band Superconducting Sample, $\gamma=0$.}

The magnetization $-4 \pi M(T)$ under fixed applied magnetic field $H=0.5$ and $H=0.7$ for $R=20 \%, 30 \%, 40 \%, 50 \%$ is shown in Fig. 2a, b. We observed that the magnetization decreases as the temperature increases, the magnetization decays in a same value $M_{c}=0.044$ for $H=0.5$ and $M_{c}=0.034$ for $H=0.7$ (called critical magnetization) to zero, independent of the surface roughness. In this critical magnetization, a first-order transition occurs from the superconducting phase to the normal state. It is observed in Fig. 2a, b that when the surface roughness becomes inhomogeneous $R \neq 0$, the critical temperature that defines the superconducting phase decreases, thus there is an entry of vortex in the sample at lower $T$ as $R$ increases.

The heat capacity $C(T)$ and magnetization with increasing temperature at a fixed external magnetic field $H=0.5$ are shown in Fig. 3a, b, respectively. In Fig. 3a, we can see a peak of heat capacity at $T=0.45$, and when the temperature increases, a first-order transition from the superconductor state to the normal state is observed in both, i.e., heat capacity and magnetization, see Fig 3b. That peak of the heat

Fig. 1 Schematic representation of the studied square with surface roughness, size $L=40 \xi_{0}$ (Color figure online)

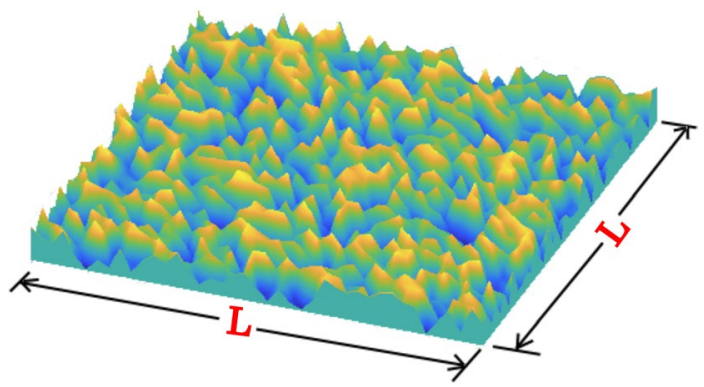


Fig. 2 Magnetization as a function of temperature at a $H=0.5$ and $\mathbf{b} H=0.7$ for $R=20 \%, 30 \%, 40 \%, 50 \%$ for a single-band superconductor. $M_{c}$ is the critical manetization (Color figure online)
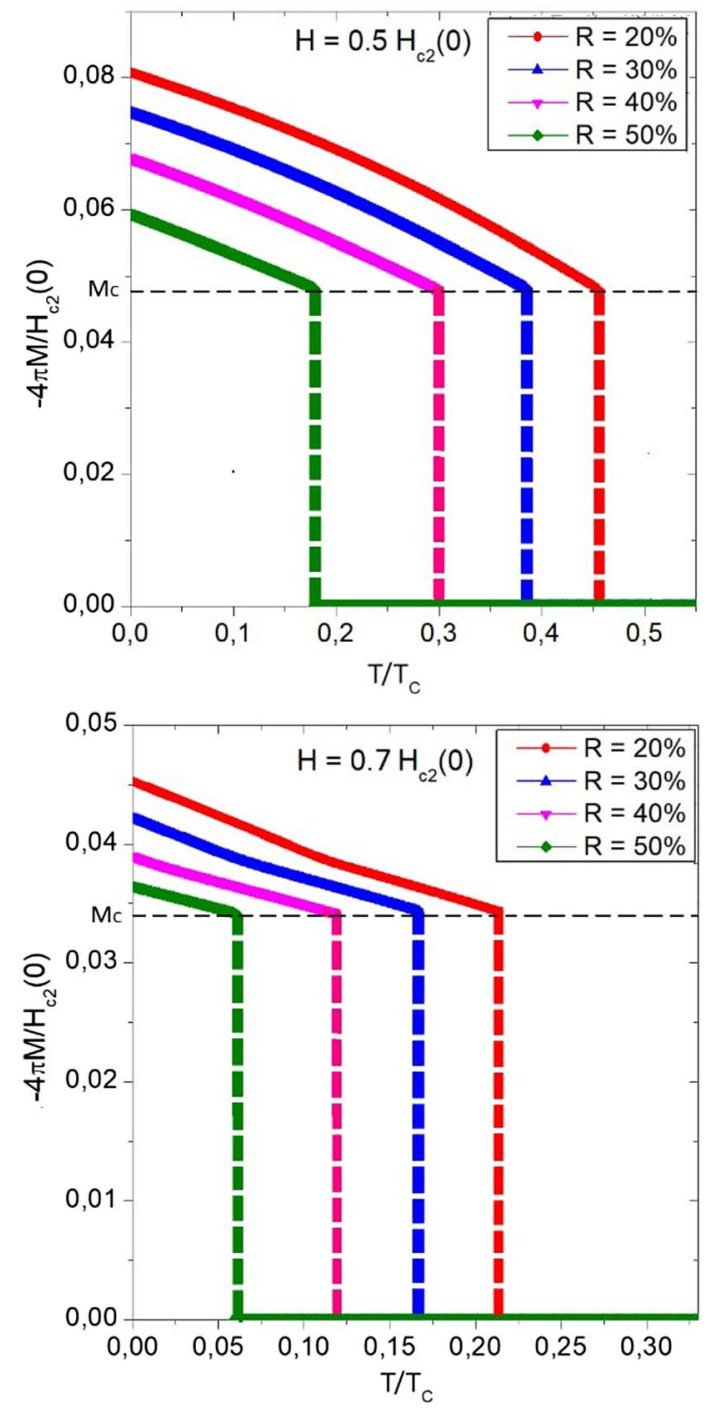

capacity is associated with the transition phase from a superconducting state to a normal state.

Now we study the effect on the heat capacity due to the variation of the surface roughness for a single-band superconductor. In Fig. 4, the dependence of heat capacity with respect to temperature is shown for three different magnetic fields, initially at (a) $H=0.5$, (b) $H=0.694$ and (c) $H=0.7$. In this Fig. 4, it is observed a maximum value of heat capacity for these three different cases and when the surface roughness is incremented shows that this first-order transition occurs in more low temperature. The transition from a superconducting state to a normal state is due to the surface energy barrier is smaller, with which the entry of vortex into the 

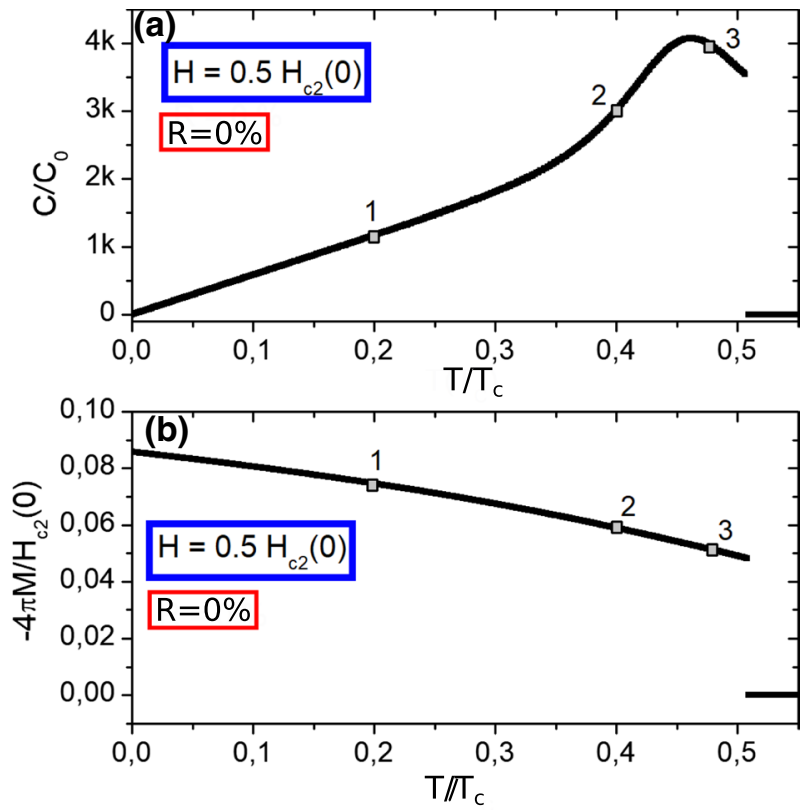

Fig. 3 a Heat capacity and $\mathbf{b}$ magnetization as function of temperature for a single-band superconductor with $R=0$ at $H=0.5$ (Color figure online)
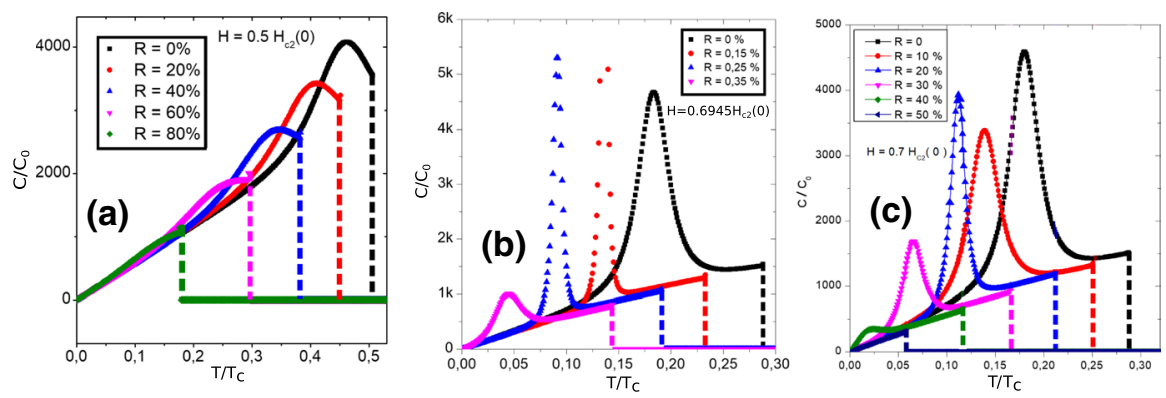

Fig. 4 Heat capacity as function of temperature at $\mathbf{a} H=0.5, \mathbf{b} H=0.6945$ and $\mathbf{c} H=0.7$, for different $R$ for a single-band sample (Color figure online)

superconductor sample is favored. In Fig. 5, the free Gibbs energy $G$ is presented for (a) $H=0.5$ and (b) $H=0.7$ for $R=0 \%, 10 \%, 20 \%, 30 \%, 40 \%, 50 \%$. It is important to observe that as the magnetic field increases under the same $R$, the free energy is minimized at a lower temperature, by means of a first-order phase transition. When $R$ increases, it is appreciated that the energy is minimized more quickly.

Finally, in Fig. 6, we present the entropy in the superconducting system, where we observe the variation in the entropy, from the superconducting state to the normal state. In Fig. 6a, b, the entropy is presented for a single-band sample 
Fig. 5 Gibbs free energy

$G$ in ZFC process, for

$R=0 \%, 10 \%, 20 \%, 30 \%, 40 \%, 50 \%$

at (a) $H=0.5$ and (b) $H=0.7$

for a single-band superconduc-

tor (Color figure online)
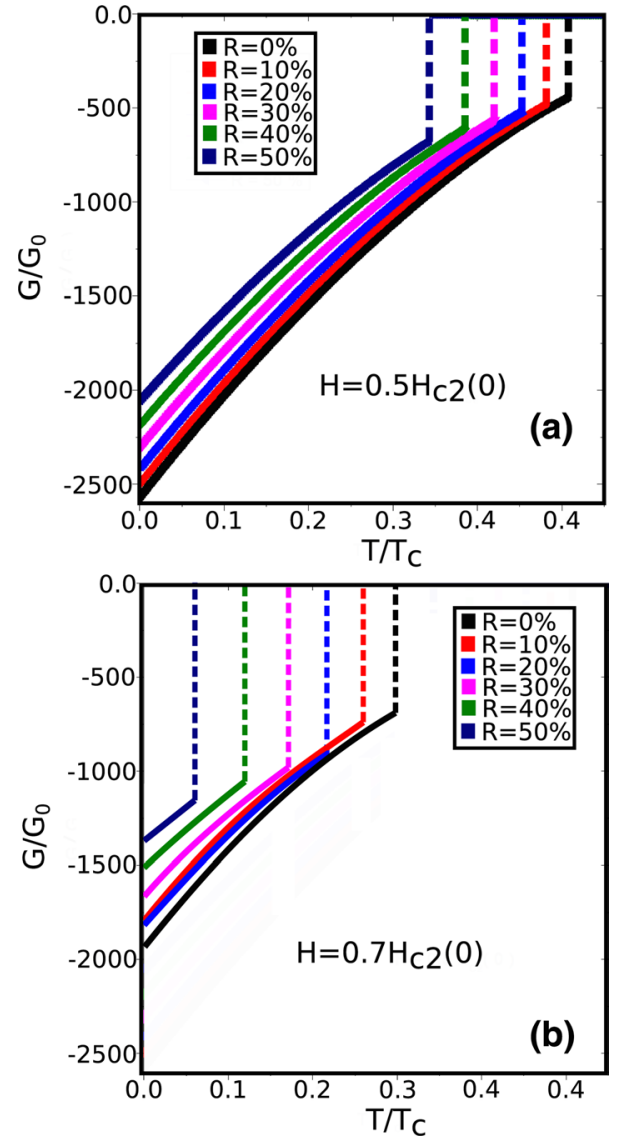

for $R=0 \%, 10 \%, 20 \%, 30 \%, 40 \%, 50 \%$, at (a) $H=0.5$ and (b) $H=0.7$. It is easy to see that as measured that the magnetic field and the surface roughness on the superconductor are increased, the entropy decides more quickly through a firstorder transition. Thus, the energy barrier decreases, and the localized loss of the normal state is favored, i.e., fluxoids in the superconducting sample.

Figure 7 a shows the vortex configuration from Fig. 3. The points (1) and (2) show the multi-vortex state at $T=0.2$ and $T=0.4$, respectively. The peak in the heat capacity near to the point $(3), T \approx 0.47$, is a giant vortex, and after this peak, the sample reaches the normal state quickly. In Fig. 7b, the vortex configuration at $H=0.694$ for several temperatures. It can be seen that at point (1), at $T=0.075$, the superconducting sample presents well-defined multi-vortex states, but when the temperature is increased, the superconductor evolves to a single giant-vortex state, as observed in points (3), at $T=0.175$ and (4), at $T=0.2$. It is easy to see that the evolution of the vortex states in the superconducting sample is directly related to the heat capacity peaks. 
Fig. 6 Entropy $S$ as func-

tion of temperature for $R=0 \%, 10 \%, 20 \%, 30 \%, 40 \%, 50 \%$

for $\mathbf{a} H=0.5$ and $\mathbf{b} H=0.7$ for a single-band superconductor (Color figure online)

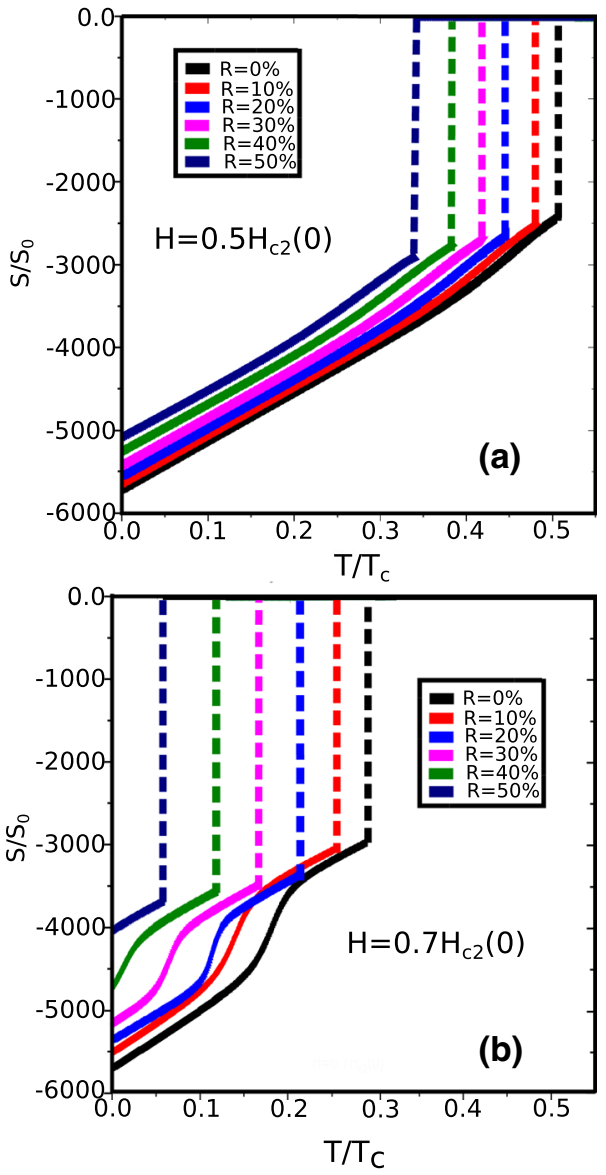

\subsection{Two-Band Superconducting Sample, $\gamma=0.01$.}

Once the different results for a single-band superconducting case have been presented and discussed, we now proceed to study the effect that surface roughness has, on the different electronic and thermodynamic variables on a two-band superconducting system, we will study the coupling between bands by means of a Josephson type coupling. To do this, we will start with the magnetization without surface roughness, i.e., isotropic and homogeneous. With this, in Fig. 8, we present the magnetization $-4 \pi M$ as a function of the temperature at $H=0.5$ for $R=20 \%, 30 \%, 40 \%, 50 \%$. As the $R$ increases, the magnetization decays at higher temperatures. These transitions in the magnetization are signals of entry of vortex in the superconductor system, this expected behavior because the energy barrier at the boundary is lower and this is responsible for the vortex ingress occurs for lower temperatures. 

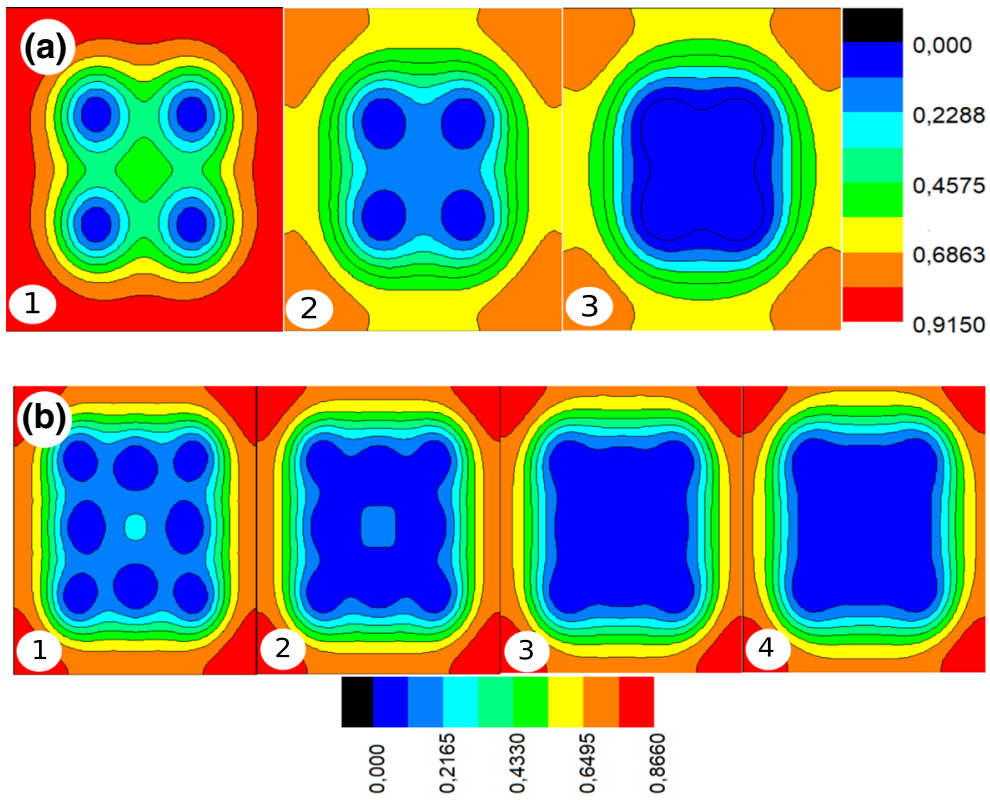

Fig. 7 Vortex state for mono-band superconductor at $R=0$. a Vortex states at $T=0.2(1), T=0.4$ (2) and $T=0.47$ (3) at magnetic field $H=0.5 H_{c 2}(0)$. b Vortex states at $T=0.015(1), T=0.125$ (2), $T=0.175$ (3) and $T=0.2$ (4) at $H=0.6945$ (Color figure online)

Fig. 8 Magnetization

$-4 \pi M$ as a function of temperature at $H=0.5$ at $R=20 \%, 30 \%, 40 \%, 50 \%$, for a two-band superconducting system (Color figure online)

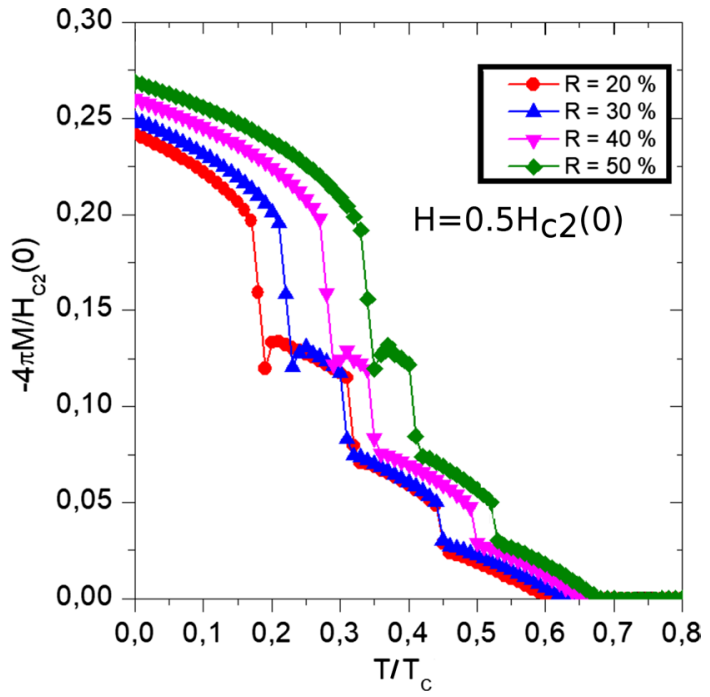

The magnetization $-4 \pi M$ as a function of the temperature for $R=10 \%, 20 \%, 30 \%, 40 \%, 50 \%$, at $H=0.9$ is shown in Fig. 9. It is easy to observe that as the roughness percentage increases, the superconductor/normal transitions 
Fig. 9 Magnetization

$-4 \pi M$ as a function of temperature at $H=0.9$, for $R=10 \%, 20 \%, 30 \%, 40 \%, 50 \%$, for a two-band superconducting system (Color figure online)

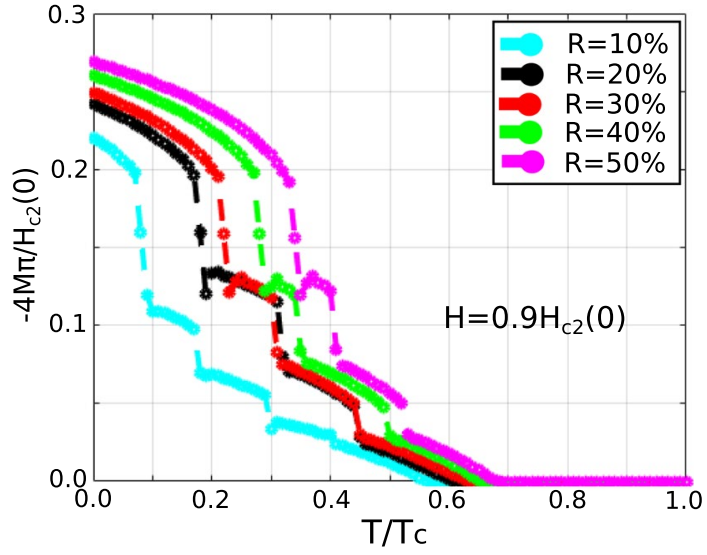

persist at higher temperatures, i.e., the critical temperature increases. Additionally, first and second-order transitions are observed at different intervals of temperature in the magnetization, which is indicative of the ingress of the vortex into the superconductor system.

In Fig. 10, we show the heat capacity $C(T)$ as a function of temperature for $R=0.5 \%, 0.4 \%, 0.3 \%, 0.2 \%, 0.1 \%, 0 \%$ at $H=0.9$. This superconducting square is composed of two interacting bands with the main band [27, 28] that interact between them through the Josephson type coupling. Narrow and well-defined heat capacity peaks are observed at specific temperatures, which is a reflection of first-order transitions in the superconductor. From the thermodynamic point of view, it is shown that the heat capacity increases with the entry of the vortex, thus establishing an addition of an enormous amount of energy, to generate an important change in temperature in the superconducting sample.

In Fig. 11, we present Gibbs free energy, for a two-bands superconducting system, at $H=0.5$ and $R=0 \%, 20 \%, 30 \%, 40 \%, 50 \%$. It is observed that as the surface roughness percentage of the superconductor is increased, the critical temperature decreases, in this way the income of vortex states in the superconducting sample.

In Fig. 12, we plot the entropy $S$ as a function of the temperature at $H=0.5$ and $R=50 \%, 40 \%, 30 \%, 20 \%, 0 \%$, for two-band superconducting system. It is easy to see that as the surface roughness percentage is increased, a phase transition from the mixed state to a normal state at lower temperatures, which favors the income of vortex states in the superconducting sample.

In Fig. 13, we present the vortex state for the superconductor of two band at (a) $H=0.5$, (b) $H=0.9$ and (c) $H=1,1$ at $T=0.1,0.2,0.3,0.4$ and $R=0$. The vortex state is plotted for each of the bands, we observe that at low temperatures (increasing $H$ ), the vortex configuration is different from the one presented in Fig. 7. However, we observe a loss in the vortex inversion symmetry $\psi(\mathbf{r}) \neq \psi(-\mathbf{r})$ for high temperatures, this is indicative of the generation of a giant vortex, the result of the superposition of the centers of different vortex in the same position, in which the repulsion 


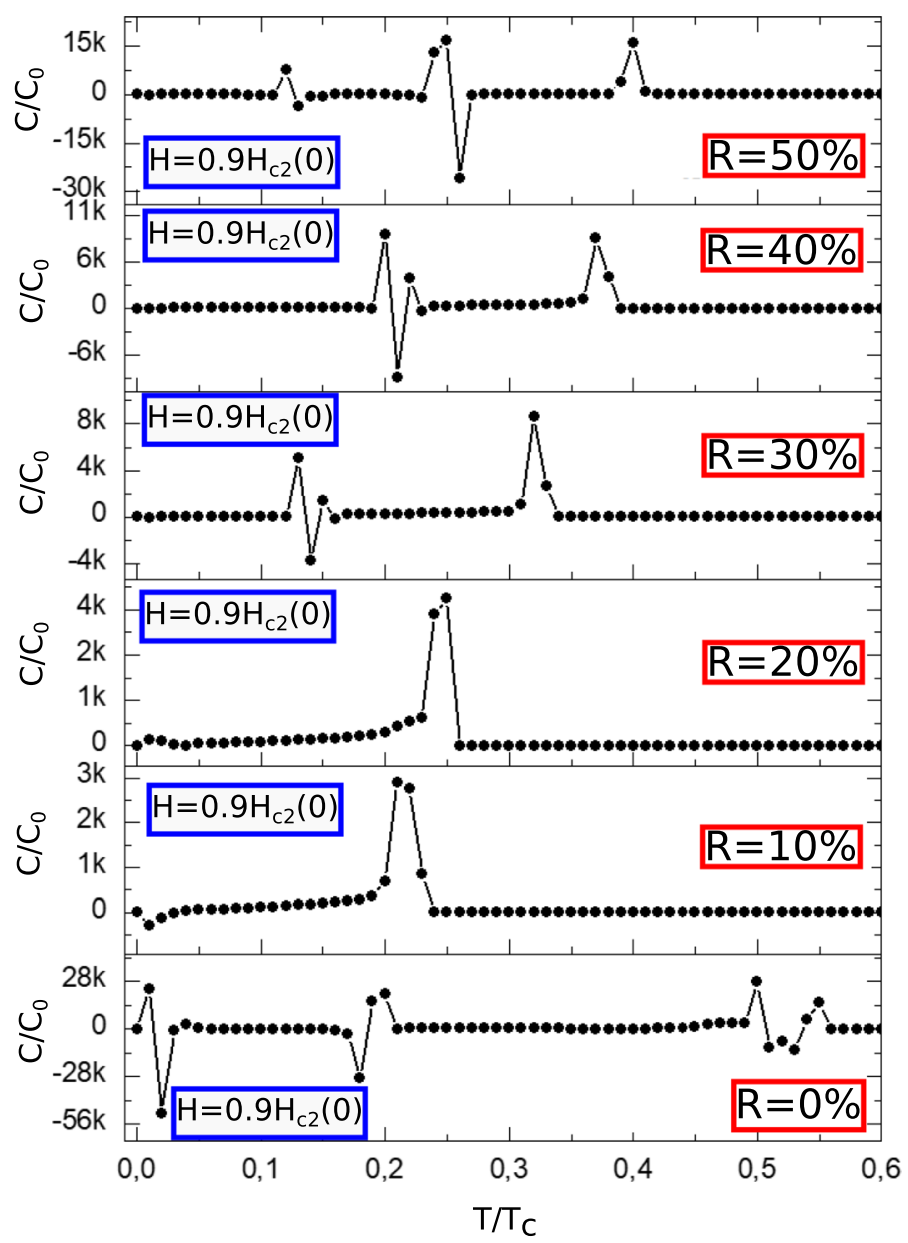

Fig. 10 Heat capacity $C(T)$ at $H=0.9$, for $R=0.5 \%, 0.4 \%, 0.3 \%, 0.2 \%, 0.1 \%, 0 \%$, for a two-band superconductor (Color figure online)

interaction between them is opaque, given by very short-range action, interaction possible only in multi-band systems.

In Fig. 14, we present the vortex state for a two-band superconducting sample with (a) $R=10 \%$, (b) $R=20 \%$, (c) $R=30 \%$ and d) $R=40 \%$, at $T=0.5,0.6,0.7,0.8$ and $H=0.5$, in a two-bands system. It is easy to observe that as $R$ increases, the system favors the stability of the superconducting states in each of the bands. Besides, when the temperature continues to increase, it is observed that there is instability in the superconducting state, which is only affected by the loss of the superconducting state in the center of the sample, this state of equilibrium occurs when all the vortex enters, and since roughness is a random variable on the surface of the sample, it makes the entry of vortex 
Fig. 11 Gibbs free energy $G$ as function of temperature for a two-band superconductor at $H=0.5$ and $R=0 \%, 20 \%, 30 \%, 40 \%, 50 \%$ (Color figure online)

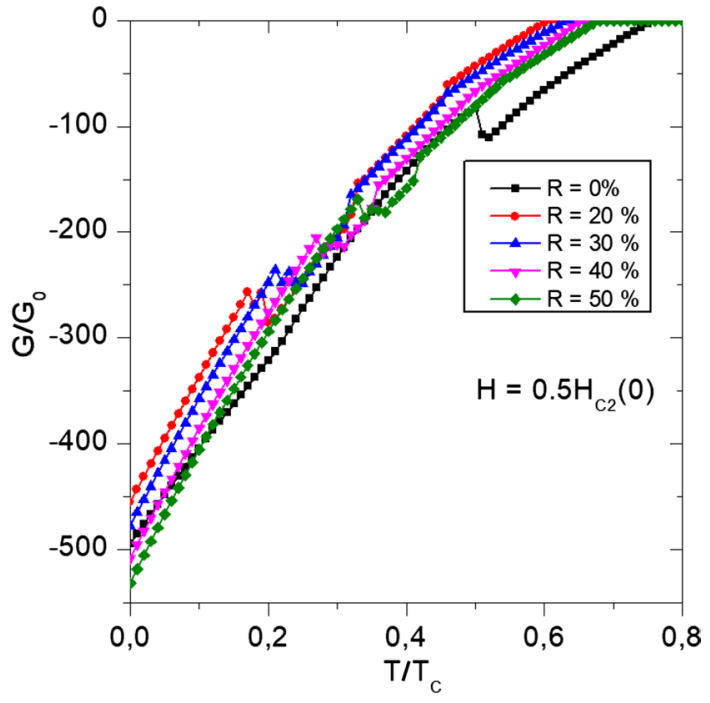

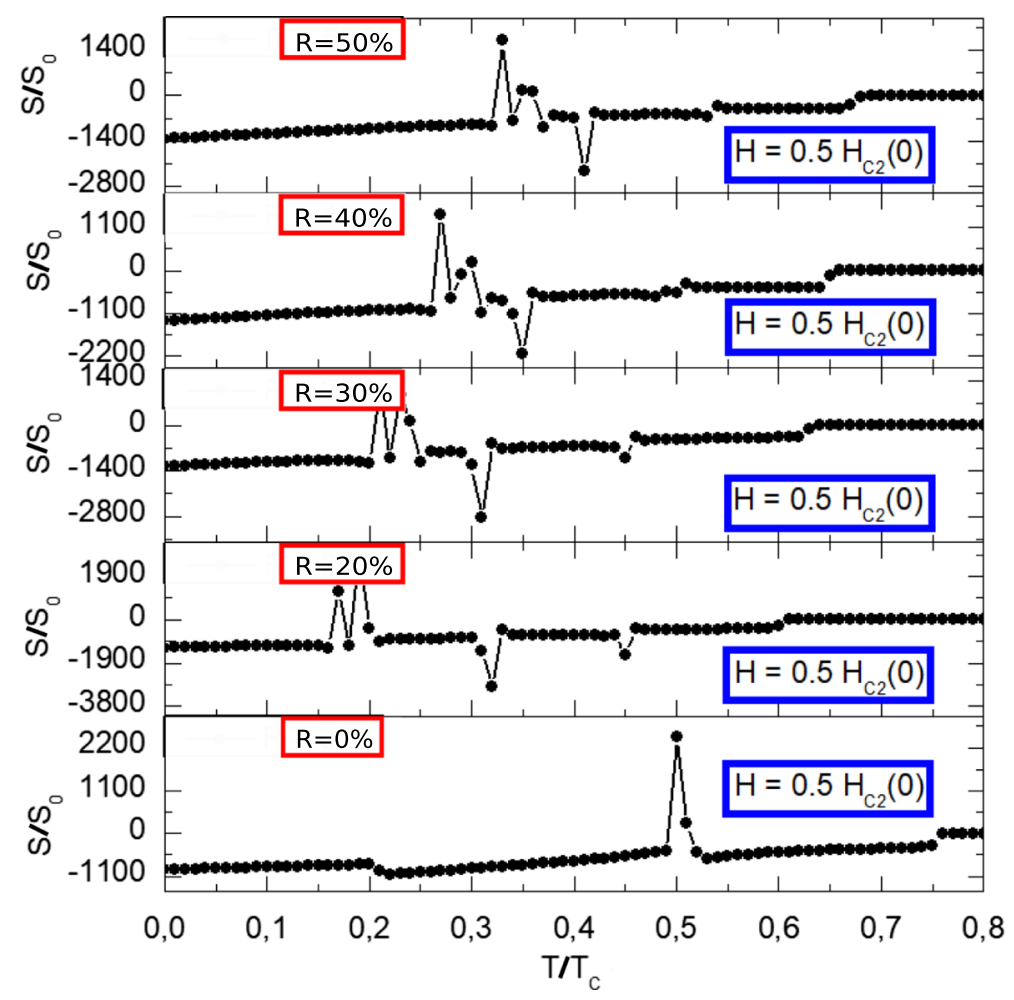

Fig. 12 Entropy $S$ as function of temperature for $R=50 \%, 40 \%, 30 \%, 20 \%, 0 \%$ at $H=0.5$ (Color figure online) 

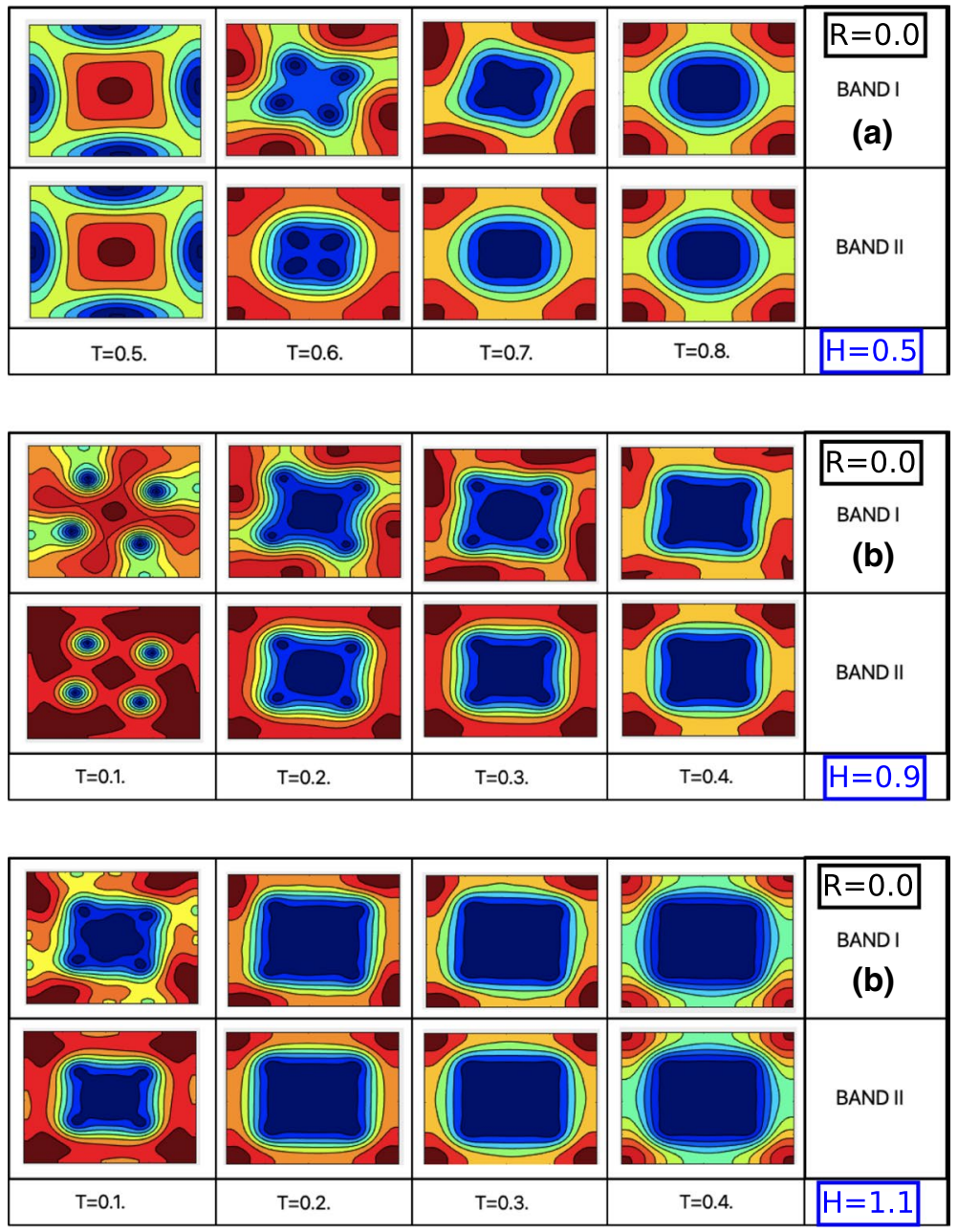

Fig. 13 Vortex states in a square superconductor at a $H=0.5$, b $H=0.9$ and c $H=1,1$ at $T=0.1,0.2,0.3,0.4$ and $R=0$ for a two-band system (Color figure online)

anomalous and the possibility of the creation of the giant vortex in the center of the sample is reinforced.

\section{Conclusions}

As summary, we have studied thermodynamic and magnetic properties such as heat capacity, Gibbs free energy, entropy in a single-band mesoscopic superconductor with roughness on its square surface through the ZFC process. Subsequently, we have extended the study to a two-band mesoscopic superconducting square with a irregular surface, in which have observed vortex states at several $H$ and $T$. It is 

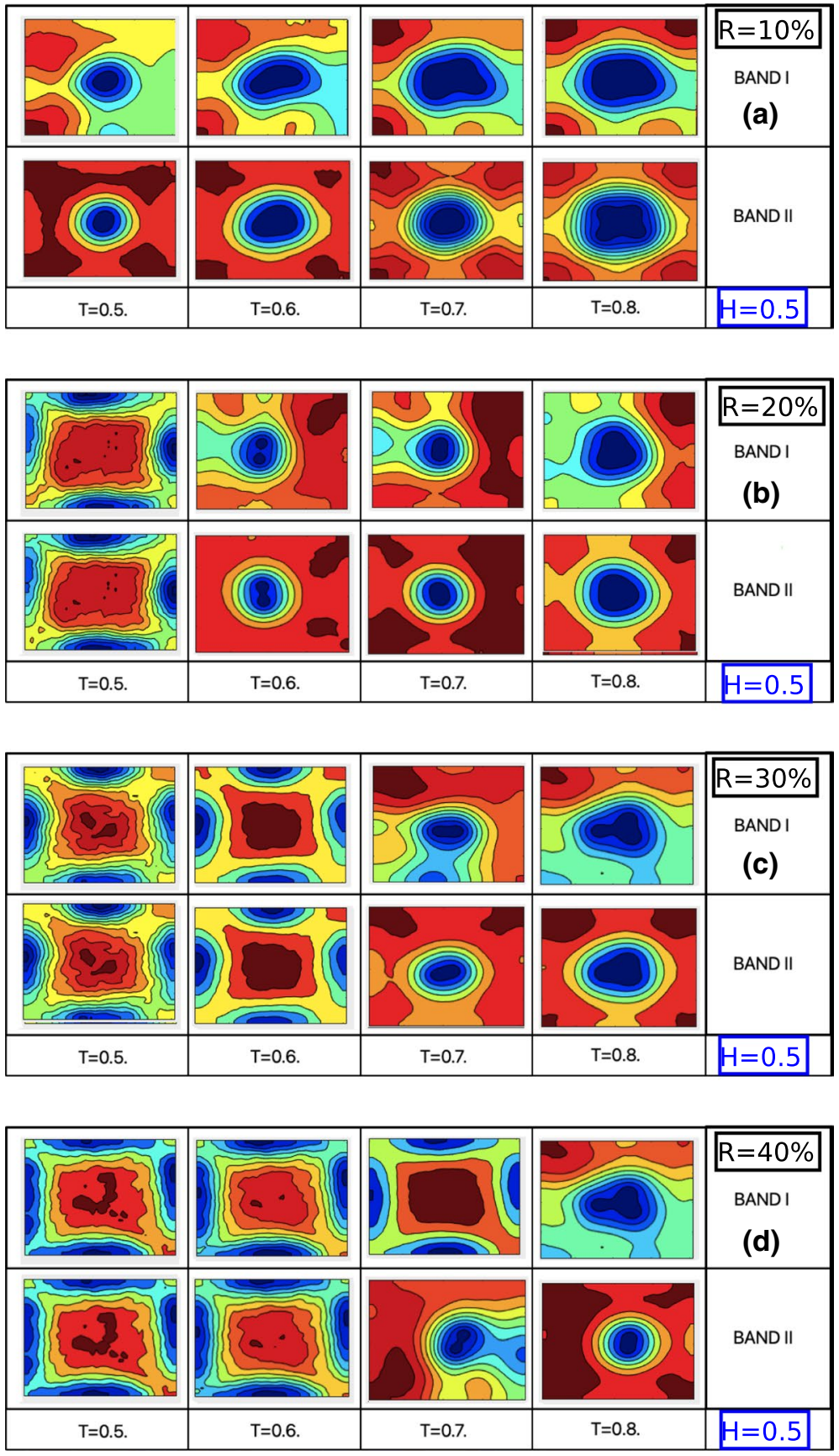

Fig. 14 Vortex states with a $R=10 \%$, b $R=20 \%$, c $R=30 \% \mathrm{~m}$ and $\mathbf{d} R=40 \%$, at $T=0.5,0.6,0.7,0.8$ and $H=0.5$, in a two-band system (Color figure online) 
observed that in both a single-band and two-band superconductor, increasing $T$ and $H$ causes an instability and saturation of the superconducting state. Also, we observed that when the imperfections on the superconductor surface increase, the $T_{c}$ increases too. Besides, the vortex states can be controlled through a cooperative variation between $H$ and $T$. Finally, we propose that the surface roughness of the superconductor can be varied by using doping that will cause localized imperfections in certain regions of the superconductor. This theory may be used for the study of multi-band superconductivity in nickelates and iron-based composts [36-39].

Acknowledgements C. A. Aguirre would like to thank the Brazilian agency CAPES, for financial support and the Ph.D. fellowship. J. Faúndez and S. G. Magalhães thank FAPERGS, CAPES and CNPq for partially financing this work under the Grant PRONEX 16/0490-0. A. Mosquera would like to thank the FONCIENCIAS 2018-Unimagdalena.

\section{References}

1. V.V. Moshchalkov, L. Gielen, C. Strunk, R. Jonckheere, X. Qiu, C. Van Haesendonck, Y. Bruynseraede, Nature 373, 319 (1995). https://doi.org/10.1038/373319a0

2. P.G. de Gennes, Superconductivity in Metals and Alloys (Addison-Wesley, Reading, MA, 1989)

3. E. Sardella, M.M. Doria, P.R.S. Netto, Phys. Rev. B. 60, 13158 (1999). https://doi.org/10.1103/ PhysRevB.60.13158

4. C.C. de Souza Silva, L.R.E. Cabral, J.A. Aguiar, Phys. Rev. B 63, 134526 (2001). https://doi.org/10. 1103/PhysRevB.63.134526

5. V.R. Misko, V.M. Fomin, J.T. Devreese, V.V. Moshchalkov, Phys. Rev. Lett. (2003). https://doi.org/ 10.1103/PhysRevLett.90.147003

6. L.R.E. Cabral, B.J. Baelus, F.M. Peeters, Phys. Rev. B (2004). https://doi.org/10.1103/PhysRevB. 70.144523

7. J. Barba-Ortega, E. Sardella, J.A. Aguiar, Phys. C 470, 1964 (2010). https://doi.org/10.1016/j.physc. 2010.08.008

8. J. Barba-Ortega, E. Sardella, J.A. Aguiar, Supercond. Sci. Technol. (2011). https://doi.org/10.1088/ 0953-2048/24/1/015001

9. B.J. Baelus, K. Kadowaki, F.M. Peeters, Phys. Rev. B (2005). https://doi.org/10.1103/PhysRevB.71. 024514

10. J. Barba-Ortega, E. Sardella, J.A. Aguiar, Phys. C 485, 107 (2012). https://doi.org/10.1016/j.physc. 2012.11.004

11. G.J. Kimmel, A. Glatz, V.M. Vinokur, I.A. Sadovskyy, Sci. Rep. 9, 1 (2019). https://doi.org/10. 1038/s41598-018-36285-4

12. P.S. Deo, J.P. Pekola, M. Manninen, Eur. Lett. 50(5), 649 (2000). https://doi.org/10.1209/epl/ i2000-00319-x

13. F.R. Ong, O. Bourgeois, Eur. Lett. 79(6), 67003 (2007). https://doi.org/10.1209/0295-5075/79/ 67003

14. X. Ben, M.V. Milosevic, F.M. Peeters, Phys. Rev. B (2010). https://doi.org/10.1103/PhysRevB.81. 064501

15. W.A. Little, R.D. Parks, Phys. Rev. Lett. 9, 9 (1962). https://doi.org/10.1103/PhysRevLett.9.9

16. R.D. Parks, W.A. Little, Phys. Rev. 133, A97 (1964). https://doi.org/10.1103/PhysRev.133.A97

17. F.R. Ong, O. Bourgeois, S.E. Skipetrov, J. Chaussy, Phys. Rev. B 74, 140503(R) (2006). https://doi. org/10.1103/PhysRevB.74.140503

18. C. Meyers, Phys. Rev. B (2003). https://doi.org/10.1103/PhysRevB.68.104522

19. J.J. Palacios, Stat. Dyn. Asp. Mesos. Syst. (2000). https://doi.org/10.1007/3-540-45557-4_21

20. P.H. Huang, C.M. Lu, Sci. World J. (2014). https://doi.org/10.1155/2014/863404

21. F. Bouquet, Y. Wang, I. Sheikin, T. Plackowski, A. Junod, S. Lee, S. Tajima, Phys. Rev. Lett. (2002). https://doi.org/10.1103/PhysRevLett.89.257001 
22. A.M. Gabovich, A.I. Voitenko, Low Temp. Phys. 28, 803 (2002). https://doi.org/10.1088/0953$8984 / 14 / 6 / 320$

23. A.M. Gabovich, A.I. Voitenko, M.S. Li, H. Szymczak, Low Temp. Phys. 28, 803 (2002). https://doi. org/10.1063/1.1528571

24. Y. Kleeorin, H. Thierschmann, A. Georges, L.W. Molenkamp, Y. Meir, Nat. Commun. 10(1), 1 (2019). https://doi.org/10.1038/s41467-019-13630-3

25. M.V. Milošević, R. Geurts, Phys. C 470, 791-795 (2010). https://doi.org/10.1016/j.physc.2010.02. 056

26. C. Aguirre, E. Sardella, J. Barba-Ortega, Solid. State. Commun. (2020). https://doi.org/10.1016/j. ssc. 2019.113799

27. T. Nunes, C. Aguirre, A. de Arruda, J. Barba, Eur. Phys. J. B 93, 69 (2020). https://doi.org/10.1140/ epjb/e2020-100418-4

28. J. Garaud, J. Carlström, E. Babaev, Phys. Rev. Lett. (2011). https://doi.org/10.1103/PhysRevLett. 107.197001

29. J. Garaud, J. Carlström, E. Babaev, M. Speight, Phys. Rev. B 87, 014507 (2013). https://doi.org/10. 1140/epjb/e2020-100418-4

30. M. Zehetmayer, M. Eisterer, J. Jun, S.M. Kazakov, J. Karpinski, A. Wisniewski, H.W. Weber, Phys. Rev. B (2002). https://doi.org/10.1103/PhysRevB.66.052505

31. C.A. Aguirre, H.D. Blas, J. Barba-Ortega, J. Low. Temp. Phys. 195, 124 (2019). https://doi.org/10. 1007/s10909-019-02147-0

32. C.A. Aguirre, H.D. Blas, J. Barba Ortega, Phys. C 554, 8 (2018). https://doi.org/10.1016/j.physc. 2018.08.010

33. M.V. Milosević, F.M. Peeters, Phys. Rev. Lett. (2004). https://doi.org/10.1103/PhysRevLett.93. 267006

34. C.D. Dewhurst, R. Cubitt, M.R. Eskildsen, S.M. Kazakov, J. Karpinski, J. Phys. C 404, 135 (2004). https://doi.org/10.1016/0921-4534(93)90777-N

35. A.C. Romaguera, S. Silva, J. Math. Phys (2013). https://doi.org/10.1063/1.4819247

36. D. Li, K. Lee, B.Y. Wang, M. Osada, S. Crossley, H.R. Lee, Y. Cui, Y. Hikita, H.Y. Hwang, Nature 572, 624 (2019). https://doi.org/10.1038/s41586-019-1496-5

37. S. Zeng, C.S. Tang, X. Yin, C. Li, M. Li, Z. Huang, J. Hu, W. Liu, G.J. Omar, H. Jani, Z.S. Lim, K. Han, D. Wan, P. Yang, S.J. Pennycook, A.T.S. Wee, A. Ariando, Phys. Rev. Lett. (2020). https://doi. org/10.1103/PhysRevLett.125.147003

38. H. Hosono, A. Yamamoto, H. Hiramatsu, Y. Ma, Mater. Today 21, 3 (2018). https://doi.org/10. 1016/j.mattod.2017.09.006

39. C.H. Wang, T.K. Chen, C.C. Chang, Y.C. Lee, M.J. Wang, K.C. Huang, P.M. Wu, M.K. Wu, Phys. C 549, 61 (2018). https://doi.org/10.1016/j.physc.2018.02.047

Publisher's Note Springer Nature remains neutral with regard to jurisdictional claims in published maps and institutional affiliations. 